\title{
Balloon Induced Dissection of Left Anterior Descending Artery and its Bidirectional Progression Involving Left Circumflex Ar- tery
}

\author{
Santosh Kumar Sinha, FACC, FAESC, FSCAI* D, Puneet Aggarwal (iD and Umeshwar Pandey
}

Department of Cardiology, LPS Institute of Cardiology, GSVM Medical College, India

*Corresponding author: Santosh Kumar Sinha, FACC, FAESC, FSCAI, Associate Professor, Department of Cardiology, LPS Institute of Cardiology, GSVM Medical College, Kanpur, Uttar Pradesh, 208002, India, Tel: +91-9670220088, Fax: +91-0512-2556199; 2556521

\begin{abstract}
Coronary artery dissection is common after balloon angioplasty, few of which may be catastrophic. Here, we report a case of a76-year-old male who had diffuse disease of proximal left anterior descending artery (LAD). LAD was wired with runthrough (Terumo, Japan) and sequentially dilated with $1.5 \times 10$ and $2 \times 10 \mathrm{~mm}$ Sapphire semicompliant balloon. After balloon dilatation, dissection at the distal edge of lesion was noticed. As stent could not be tracked and pushed along the lesion as guide catheter used to back out, the guide catheter was changed to extra backup (EBU). This time, wire was seen into the false lumen along with a long segment spiral dissection spreading downward and occluding the circumflex proximally as left circumflex artery was not visible. Dissection of LAD was first sealed with 2.75 $\times 38 \mathrm{~mm}$ Xience Prime everolimus eluting stent. LCx was carefully wired using Sion wire and stented with $2.75 \times 23$ $\mathrm{mm}$ Xience Prime stent, thereby sealing the dissection and restoring LCx. Sion wire in LAD was carefully manuvoured and parked distally into true lumen. The dissection flap was still visible starting from the distal edge of the deployed stent which was sealed with another $2.5 \times 28 \mathrm{~mm}$ Xience Prime stent achieving TIMI III flow in both LAD and LCX.
\end{abstract}

\section{Keywords}

Coronary artery dissection, Balloon angioplasty, Bi-directional dissection

\section{Introduction}

Angioplasty balloon come in different diameters and are made up of diverse materials. Once inflated, the mechanism of acute lumen gain after plain balloon angioplasty in calcified lesion is local and limited dissection whereas it is plaque compression and vessel expansion in a fibrotic lesion. Coronary artery dissection is common after balloon angioplasty. This is angiographically visible in $20-45 \%$ of cases following balloon angioplasty and present in up to $85 \%$ of cases when assessed by intravascular ultrasound (IVUS) [1]. If further angioplasty of the lesion is not undertaken, then it is recognised that nearly all angiographic dissection will heal over a six month time frame. At times, few dissections are very ugly which can be catastrophic.

\section{Case Report}

A 76-year-old male, smoker and hypertensive presented with chronic stable angina- Canadian Cardiovascular Society (CCS) class III. Coronary angiography was performed after proper consent which revealed diffuse disease of proximal left anterior descending artery (LAD) with significant stenosis. Aortic sinus was little dilated, so little difficulty was encountered in cannulating left main with Judkins left (JL) guiding catheter. Lesion was wired with runthrough (Terumo, Japan) and sequentially dilated with $1.5 \times 10$ and $2 \times 10 \mathrm{~mm}$ Sapphire semicompliant balloon (Orbus Neisch, Netherland) (Figure 1A). After balloon dilatation, dissection at the distal edge was noticed (Figure 1B). As stent could not be tracked and pushed along the lesion as guide catheter used to back out, it was decided disease to change the guide catheter to extra backup (EBU) guide catheter. We could not pass the wire across the lesion

Citation: Sinha SK, Aggarwal P, Pandey U (2020) Balloon Induced Dissection of Left Anterior Descending Artery and its Bidirectional Progression Involving Left Circumflex Artery. Int J Clin Cardiol 7:209. doi. org/10.23937/2378-2951/1410209

Accepted: December 10, 2020: Published: December 12, 2020

Copyright: (C) 2020 Sinha SK, et al. This is an open-access article distributed under the terms of the Creative Commons Attribution License, which permits unrestricted use, distribution, and reproduction in any medium, provided the original author and source are credited. 


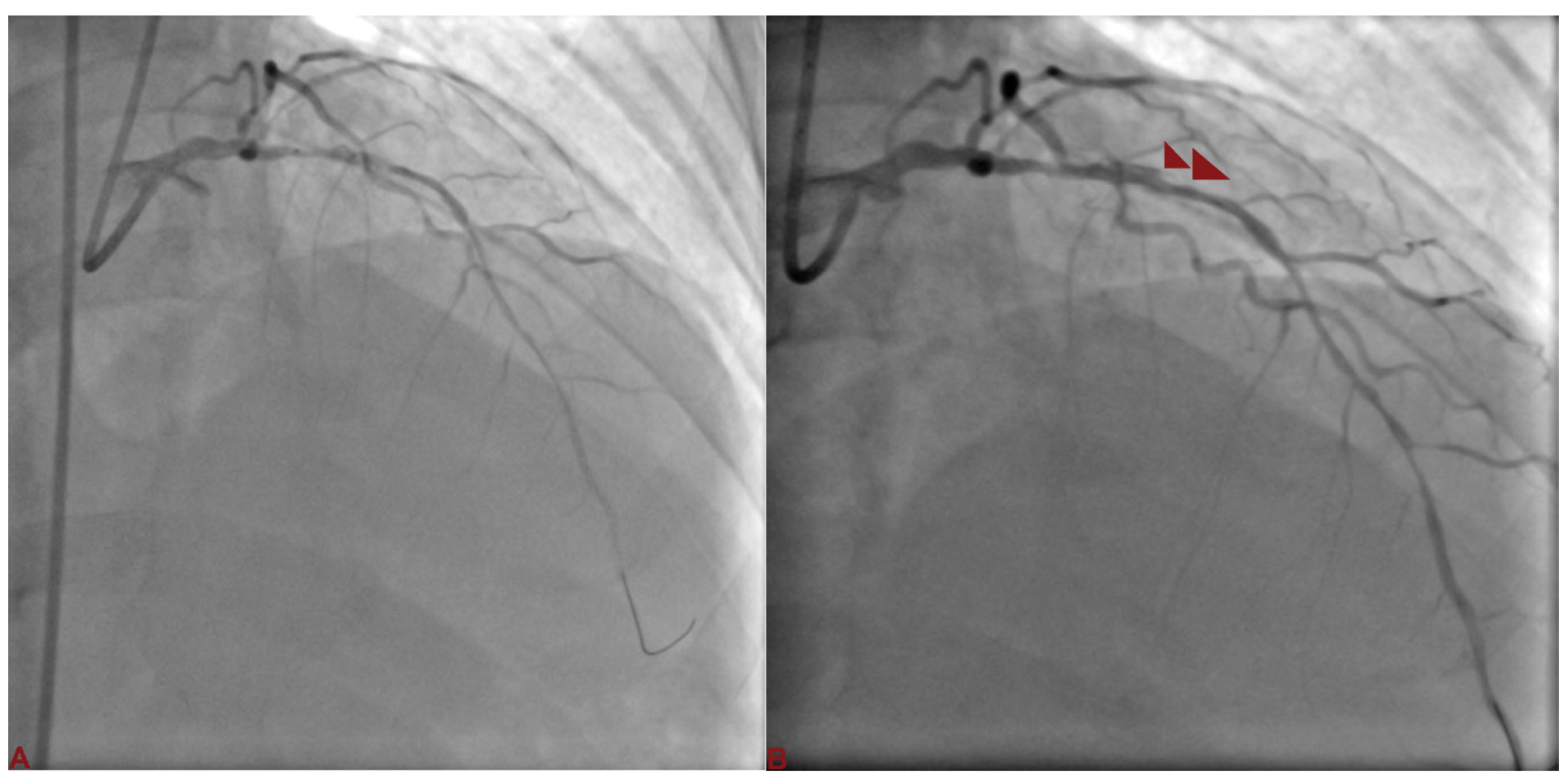

Figure 1: A) Left anterior descending artery (LAD) was wired with runthrough and sequentially dilated with $1.5 \times 10$ and $2 \times$ $10 \mathrm{~mm}$ semicompliant balloon; B) Dissection (red arrowhead) at the distal edge following balloon dilatation.

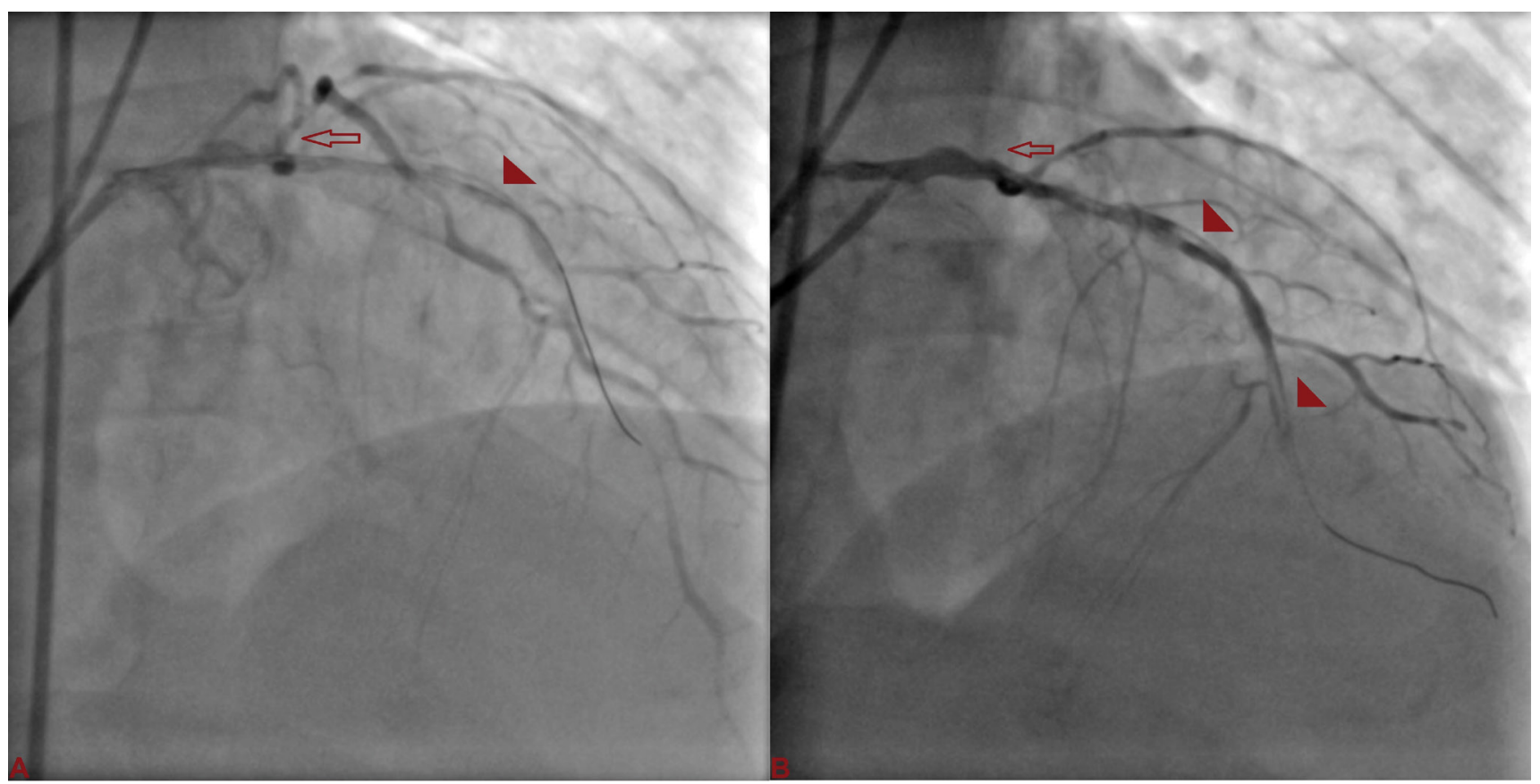

Figure 2: A) Wire was seen into the false lumen along with a long segment spiral dissection (red arrowhead) spreading downward; B) The left circumflex artery (arrow) was not visible.

and park it distally. Patient started complaining of chest pain. On contrast injection, our wire was seen into the false lumen along with a long segment spiral dissection spreading downward (Figure 2A) and the left circumflex artery was not visible (Figure 2B). It was inferred that spiral dissection was spreading bi-directionally. Keeping runthrough wire there, Sion black (Ashahi, Japan) was tried to be parked into true lumen using parallel wire technique. Once Sion wire was in true lumen distally, runthrough wire was pulled out. It was decided to seal the dissection first. $2.75 \times 38 \mathrm{~mm}$ Xience Prime everolimus eluting stent (Abott Vasc, USA) was deployed at 12 atm pressure to seal the flap (Figure $3 \mathrm{~A}$ and Figure 3B). LCx was carefully wired using Sion wire, and dilated with $1.5 \times 10 \mathrm{~mm}$ Sapphire balloon restoring the flow (Figure $4 \mathrm{~A}$ ). It was stented with $2.75 \times 23 \mathrm{~mm}$ Xience Prime stent at $12 \mathrm{~atm}$ pressure, thereby sealing the dissection and restoring LCX (Figure 4B and Figure 4C). Sion wire in $L A D$ was carefully manuvoured and parked distally into true lumen (Figure 5A, Figure 5B and Figure 5C). The dissection flap was still visible starting from the distal edge of the deployed stent. Another $2.5 \times 28 \mathrm{~mm}$ Xience Prime stent was deployed overlapping with proximal stent (Figure 6A). The Stent was post dilated by $2.75 \times$ 


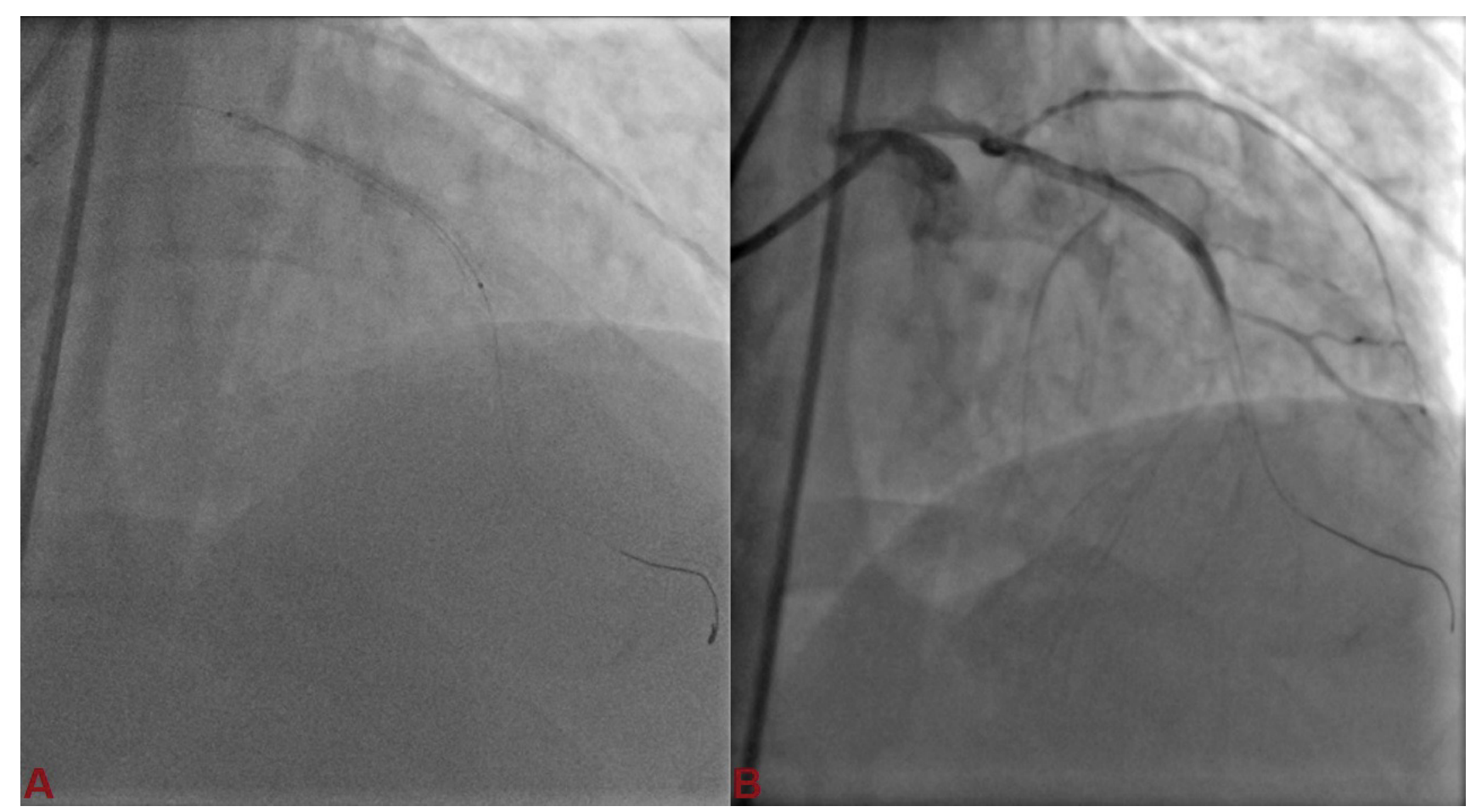

Figure 3: LAD was stented with $2.75 \times 38 \mathrm{~mm}$ Xience Prime everolimus eluting stent $(A, B)$.

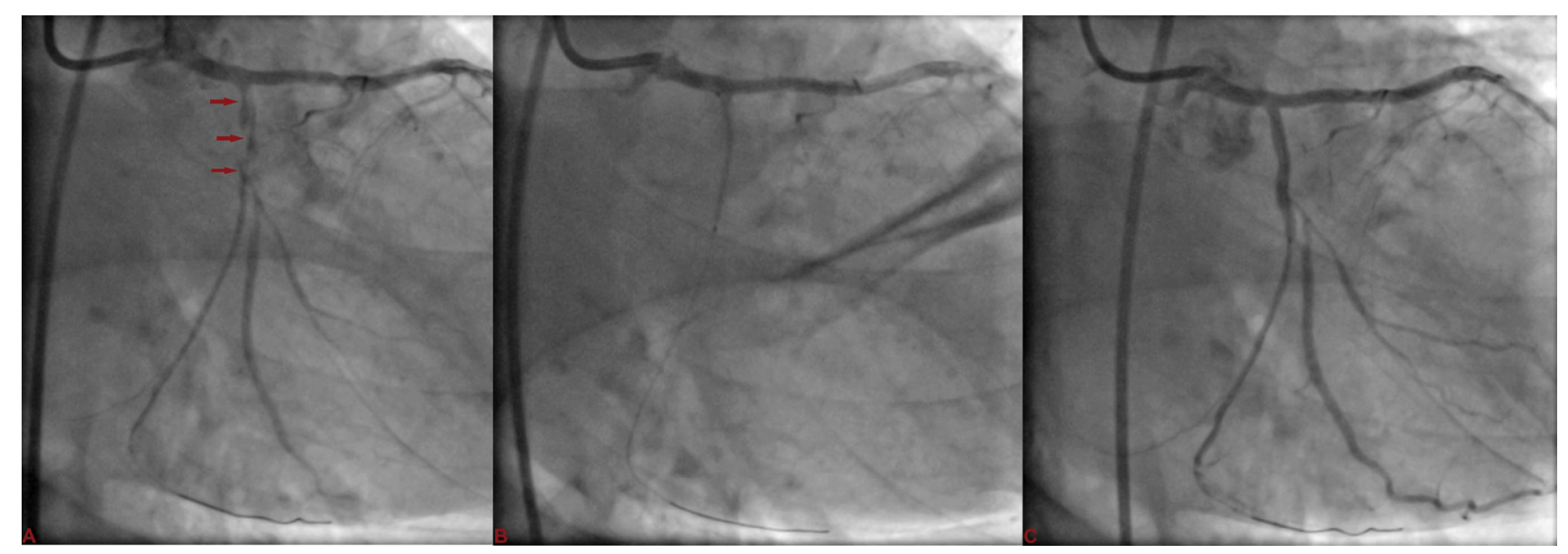

Figure 4: A) LCx was carefully wired using Sion wire, and dilated with $1.5 \times 10 \mathrm{~mm}$ Sapphire balloon restoring the flow; B) It was stented with $2.75 \times 23 \mathrm{~mm}$ Xience Prime stent at 12 atm pressure; C) thereby sealing the dissection and restoring LCx.
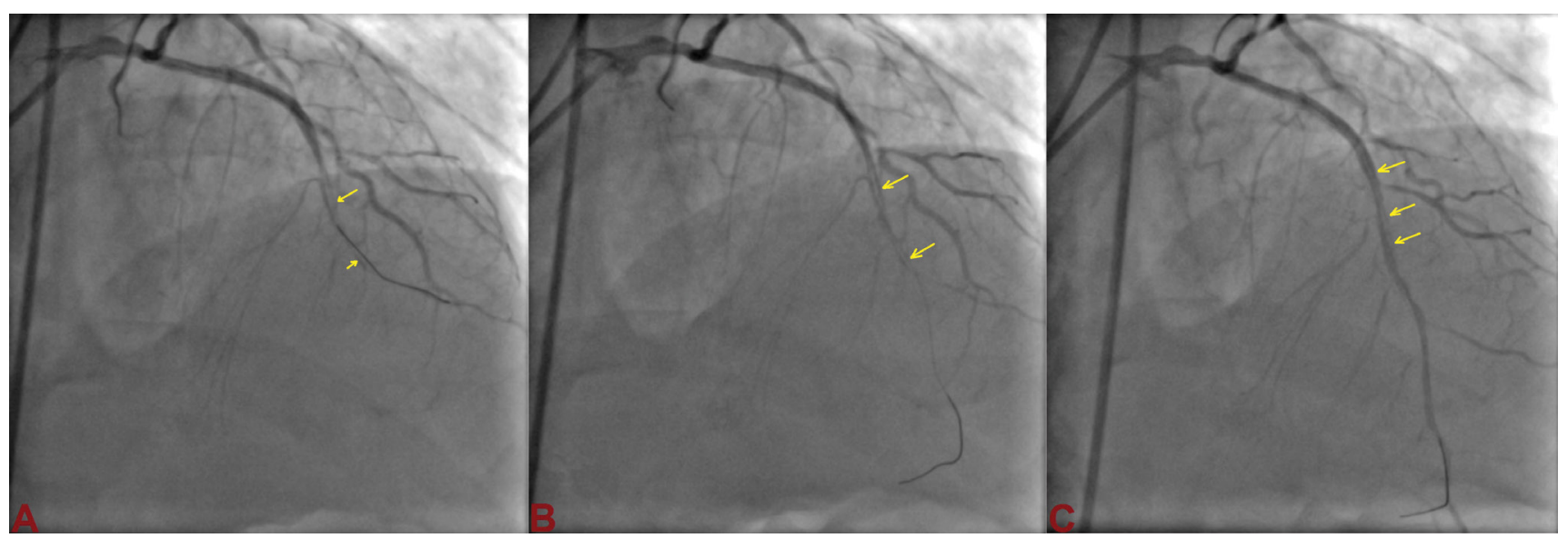

Figure 5: Sion wire in LAD was carefully manuvoured through dissection flap (arrow) and parked distally into true lumen $(A, B, C)$. 


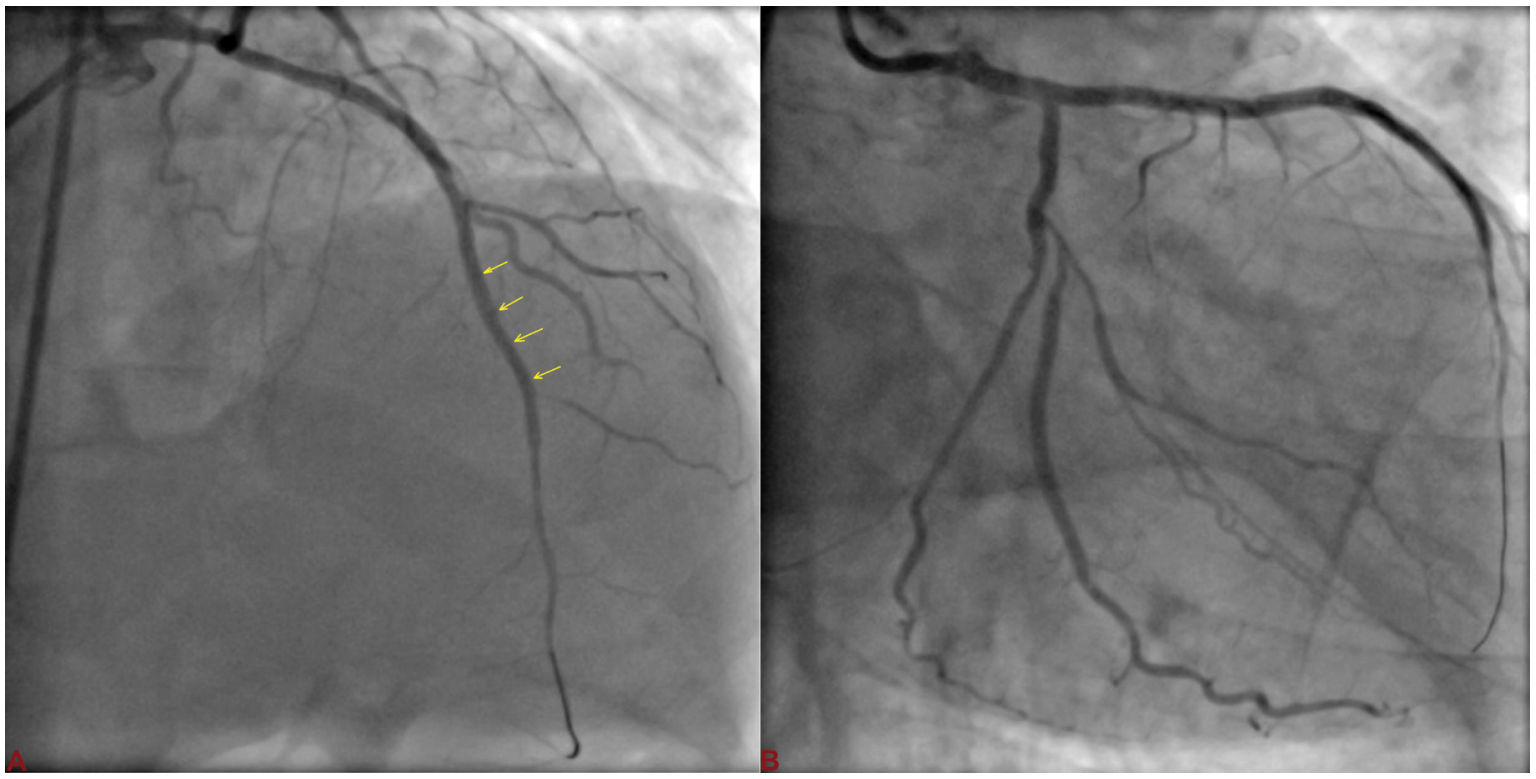

Figure 6: A) Another $2.5 \times 28 \mathrm{~mm}$ Xience Prime stent was deployed overlapping with proximal stent; B) Both the LAD and LCX started showing TIMI III flow after stenting with complete sealing of dissection flap.

$10 \mathrm{~mm}$ Sapphire non-compliant (NC) balloon. Both the LAD and LCX started showing TIMI III flow after stenting with complete sealing of dissection flap (Figure 6B). He was discharged in stable condition with prasugrel 10 $\mathrm{mg}$, aspirin- $75 \mathrm{mg}$, rosuvastatin- $40 \mathrm{mg}$, metoprolol-100 $\mathrm{mg}$, and ramipril-10 $\mathrm{mg}$.

\section{Discussion}

Balloon-induced dissection is not infrequent but may become a life-threatening complication, which may result into acute vessel closure, myocardial infarction, and sudden cardiac death [2]. The incidence of catheter-induced dissection with reported incidence of $0.02 \%$ $0.07 \%$, is most frequent caused by catheter trauma and balloon inflation $[3,4]$. It results from over dilation (balloon length > stent length or balloon: artery ratio $>1$ ), dilatation of a calcified plaque, stent strut fracture, and from aggressive handling of rigid or hydrophilic guide wires. With the advent of more complex percutaneous interventions, this complication may become more prevalent. Once coronary dissection is established, its propagation may be fast. The propagation of the dissection is mostly antegrade because of blood flow and forceful contrast injection. The left main appears to be more resistant than right coronary artery to retrograde dissection because the periostial wall and sinotubular junction of the left coronary artery are formed by more smooth muscle cells and by a dense matrix of collagen type-I fibres [3]. In our case, balloon induced dissection was bi-directional causing acute vessel closure of circumflex artery. Injection of contrast medium was another aggravating factor as the guiding catheter was not coaxial as a result of which, we had to inject contrast vigorously. The management of such dissection depends on the patency of the distal vessel and the extent of the propagation of the dissection. If there is a compromise of the distal artery bed, such as the acute closure of the artery, urgent revascularization is mandated to prevent the infarction of that myocardial area. Sometimes, such dissection can engulf the left main coronary artery and rarely aorta as well. Surgery, including aortic repair and coronary artery bypass graft surgery, has been selected in cases of extensive aortic dissection [4]. Aortic involvement has been reported mainly in case of right coronary artery dissection. Localized aortic dissection carries an excellent prognosis with the adoption of a conservative approach [5]. When a coronary artery is involved as an entry point, it usually can be safely sealed by stent implantation with good long-term outcomes. Most important point is that one should always keep wire in distal arterial bed as it will act as a rail road for all the devices including balloon and stents.

\section{Conflict of Interest}

None.

\section{Sources of Support (if applicable)}

Disclosure of funding received for this work from any of the following organizations: National Institutes of $\mathrm{He}$ alth (NIH); Welcome Trust; and other(s) - None.

\section{Statement of Equal Authors' Contribution}

All authors made equal contribution.

\section{References}

1. Hermans WR, Rensing BJ, Foley DP, Deckers JW, Rutsch W, et al. (1992) Therapeutic dissection after successful coronary balloon angioplasty: No influence on restenosis or on clinical outcome in 693 patients. J Am Coll Cardiol 20: 767-780.

2. Wykrzykowska JJ, Carrozza J, Laham RJ (2006) Aortocoronary dissection with acute left main artery occlusion: Successful treatment with emergent stenting. J Invasive Cardiol 18: E217-E220. 
3. Yip HK, Wu CJ, Yeh KH, Hang CL, Fang CY, et al. (2001) Unusual complication of retrograde dissection to the coronary sinus of valsalva during percutaneous revascularizatiuon: A single-center experience and literature review. Chest 119: 493-501.

4. Dunning DW, Kahn JK, Hawkins ET, O'Neill WW (2000) latrogenic coronary artery dissections extending into and involving the aortic root. Catheter Cardiovasc Interv 51: 387-393.

5. Shah P, Bajaj S, Shamoon F (2016) Aortic dissection caused by percutaneous coronary intervention: 2 new case reports and detailed analysis of 86 previous cases. Tex Heart Inst J 43: 52-60. 